\title{
Formation Mechanism of Amorphous Silicon Nanoparticles Synthesized by Induction Thermal Plasma
}

\author{
Xiaoyu Zhang, Kentaro Yamano, Ririko Hayashida, Hirotaka Sone, Manabu TANAKA, and Takayuki Watanabe* \\ Department of Chemical Engineering, Kyushu University, 744 Motooka, Nishi-ku, Fukuoka 819-0395, Japan
}

\begin{abstract}
This study focus on the synthesis of amorphous silicon nanoparticles and understanding the formation mechanism. Counter-flow quenching gases with different flow rates were injected from downstream of the torch to understand the effect of quenching gas on the formation of silicon nanoparticles. Transmission electron microscopy show that nanoparticles with spherical shape and agglomerates consist of smaller particles were synthesized. X-ray diffraction analysis is used to calculate the amorphization degree, which is defined as fraction of amorphous silicon in the silicon nanoparticles including both crystal and amorphous. The obtained results show that higher quenching gas flow rate leads to smaller diameter with higher amorphization degree. Electron diffraction patterns reveal that nanoparticles with diameter less than $10 \mathrm{~nm}$ are amorphous and agglomerated together, while for the nanoparticles with diameter larger than $10 \mathrm{~nm}$ are crystal. The formation mechanism of amorphous silicon nanoparticles is explained by estimated nucleation temperature and experimental results. Consequently, silicon nucleates at about $2400 \mathrm{~K}$ and then silicon vapor condenses on the nucleus. Finally, smaller nanoparticles will keep amorphous phase, while nanoparticles with a larger diameter grow to form crystalline.
\end{abstract}

\section{Introduction}

In the last 20 years, lithium ion batteries (LIB) have been widely used in small electronic devices for their advantages in energy density and cyclic life. However, with the development of electric vehicles, higher requirements have been proposed on the performance of LIB, especially on their energy density.

Carbon anodes have been proved very useful because of their stable cycling characteristics, but the small charge storage capacity also limit the development of LIB. Therefore, researches on the higher capacity anode materials have attracted great interests. Silicon is found to be a promising material because of its high charge storage capacity of $4200 \mathrm{mAh} / \mathrm{g}$ which is approximately 10 times higher than graphite as an anode material.

However, crystalline silicon undergoes a $400 \%$ volume expansion during lithiation and delithiation process and leads to serious electrode failure mechanisms. Several approaches have been proposed to overcome this problem. One of them is to form nanostructures, such as nanocrystals, nanowires, nanotubes and nanoparticles. Researchers found the particle-size-dependent fracture behavior of silicon nanoparticle during the first lithiation; that is, there exists a critical particle size of $\sim 150 \mathrm{~nm}$ below which cracking did not occur (Liu et al., 2012). Furthermore, another approach to avoid the failure of the anode material is amorphization. Amorphous silicon has a better tolerance over crystalline silicon to volume expansion processes (NG et al., 2006). Therefore, the amorphous silicon nanoparticle could be a good choice for the LIB anode.

Induction thermal plasma is characterized by high temperature, high chemical reactivity, and especially long residence times of 10-100 ms compared with other forms of thermal plasma (Tanaka et al., 2016). Furthermore, induction thermal plasma can be used to prepare amorphous material because the rapid quenching in the plasma flame tail. In this research, induction thermal plasma is used to synthesize the amorphous silicon nanoparticles, and formation mechanism is investigated.

\section{Experimental Methods}

\subsection{Experimental Setup and Conditions}

The experiment is carried out with the apparatus shown in Figure 1, which mainly consists of three parts, RF torch, reaction chamber, and particle filter. The plasma was generated by the RF power supply of $4 \mathrm{MHz}$ at $20 \mathrm{~kW}$. Experimental conditions are shown in Table 1.

Silicon powders with an average diameter around 5 $\mu \mathrm{m}$ were injected into torch through the powder feeder by Ar carrier gas. The powder feed rate was fixed at 250 $\mathrm{mg} / \mathrm{min}$. Raw materials were injected into the top of the plasma torch and immediately evaporated in the high temperature region. Silicon vapor nucleates and condenses in the tail region of the plasma because of the temperature gradient. Obtained nanoparticles were

* Corresponding author: watanabe@chem-eng.kyushu-u.ac.jp 
oxidized slowly under Ar atmosphere with $2 \mathrm{vol} \%$ of $\mathrm{O}_{2}$ after plasma experiments.

Table 1. Experimental conditions for the induction thermal plasma system

\begin{tabular}{cc}
\hline Plasma power & $20 \mathrm{~kW}$ \\
\hline Pressure & $1 \mathrm{~atm}$ \\
Frequency & $4 \mathrm{MHz}$ \\
Sheath gas & $\mathrm{Ar} 60 \mathrm{~L} / \mathrm{min}$ \\
Inner gas & $\mathrm{Ar} 5 \mathrm{~L} / \mathrm{min}$ \\
Carrier gas & $\mathrm{Ar} 3 \mathrm{~L} / \mathrm{min}$ \\
Quenching gas & $\mathrm{Ar}$ \\
Quenching gas flow rate & $0-70 \mathrm{~L} / \mathrm{min}$ \\
Powder feed rate & $250 \mathrm{mg} / \mathrm{min}$ \\
\hline
\end{tabular}

A counter flow Ar gas was injected into chamber to enhance the quenching effect for silicon nanoparticles. The range of flow rate is determined to vary from 0 to 70 $\mathrm{L} / \mathrm{min}$. The distance between the outlet of the quenching tube and the torch was fixed at $190 \mathrm{~mm}$.

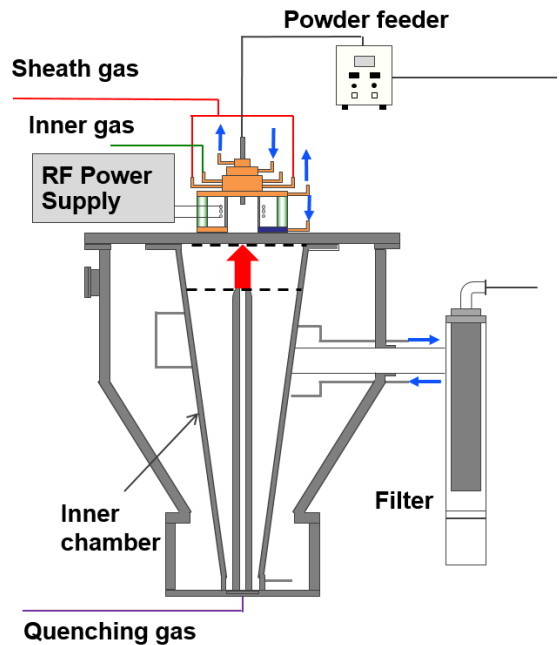

Figure 1. Experimental setup of RF induction thermal plasma for preparation of nanoparticles

\subsection{Analysis}

The crystal structure of the synthesized nanoparticles was determined through powders X-ray diffraction (XRD, Rigaku Multiflex), operating with a $\mathrm{Cu} \mathrm{K} \alpha$ source $(\lambda=$ $0.1541 \mathrm{~nm}$ ). The diffraction data was collected using the continuous scan mode at a speed of $2 \%$ min in the region of $10-90^{\circ}$ with a step of $0.04^{\circ}$. The accelerating voltage and applied current were $40 \mathrm{kV}$ and $50 \mathrm{~mA}$, respectively. The particle morphology was observed by transmission electron microscopy (TEM, JEOL JEM-2100HCKM) and size distributions were measured by counting approximately 200 different particles. Electron diffraction patterns of nanoparticles were also analyzed by TEM. In addition, amorphization degree defined as the fraction of amorphous silicon in the silicon nanoparticles including both crystal and amorphous, and was calculated by internal standard method with XRD results, while $\mathrm{ZnO}$ was chose as the standard material.

\section{Results}

Several experiments were conducted with different quenching gas flow rates, in order to understand the effect of quenching gas on amorphous silicon nanoparticles synthesized with induction thermal plasma.

\subsection{Effect of the quenching gas flow rate on the morphology of nanoparticles}

The morphology and the size distribution of the prepared nanoparticles without quenching gas are shown in Figure 2. Most of the obtained nanoparticles are spherical and have different diameters. The size distribution of particles ranges from $10 \mathrm{~nm}$ to hundreds of nanometers. The number mean diameter of the prepared silicon nanoparticles is estimated as $93 \mathrm{~nm}$ in this case.
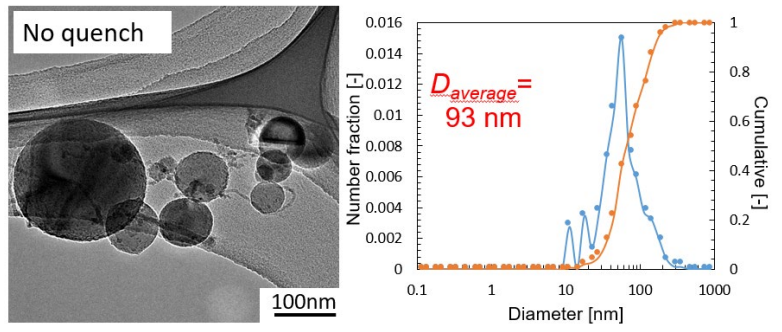

Figure 2. TEM image and size distribution of synthesized nanoparticles without quenching gas
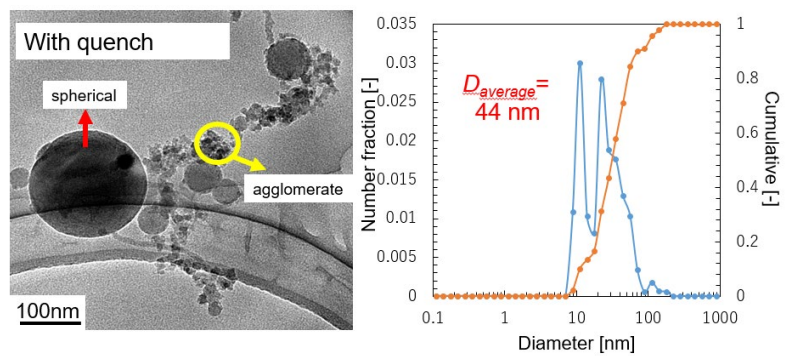

Figure 3. TEM image and size distribution of synthesized nanoparticles with quenching gas at $70 \mathrm{~L} / \mathrm{min}$

Size distribution of particles synthesized with the quenching gas injection was analyzed and shown in Figure 3. The morphology for the most nanoparticles are similar with that shown in Figure 2, that most particles are spherical. However, a large amount of nanoparticles with diameter smaller than $10 \mathrm{~nm}$ appears, while this phenomenon cannot be observed in the case without quenching gas. The agglomerated small particles are signed by yellow arrow shown in Figure 3, which has a significantly different morphology from the spherical nanoparticles. The difference between those two kinds of nanoparticles will be discussed deeply in next section.

The average diameter in Figure 3 was estimated as 44 $\mathrm{nm}$, and is smaller than that shown in Figure 2. This is because of the appearance of large amount of small particles, which can be observed from the left shift of the size distribution curve compared with Figure 2 and 3. This phenomenon can also be observed with quenching gas flow rate increases from 0 to $70 \mathrm{~L} / \mathrm{min}$, that larger amount of small particles appears with quenching gas flow rate 
increases. The reason for this phenomenon can be explained as more quenching gas leads to steeper temperature gradient. Shigeta and Watanabe (2008) revealed the quenching rate changes with different quenching gas flow rates. Quenching rate in our experimental setup was estimated as $1.0 \times 10^{4} \mathrm{~K} / \mathrm{s}$ without additional quenching gas, and it increases to $4.2 \times 10^{6} \mathrm{~K} / \mathrm{s}$ when the quenching gas flow rate increases to $20 \mathrm{~L} / \mathrm{min}$. Then the nucleation is promoted and the number of the produced nuclei increases. The vapor consumption per each nucleus becomes smaller because the total amount of silicon vapor is constant. Then a larger fraction of small nanoparticles synthesized.

\subsection{Effect of the quenching gas flow rate on the amorphization degree}

Figure 4 indicates the effect of quenching flow rate on the amorphization degree, which was defined and estimated as shown in the following equation.

$$
(\text { Amorphization Degree })=\frac{x_{a}}{x_{a}+x_{c}}
$$

where $x$ indicates the fraction of the considered species in the prepared nanoparticles. Subscripts of $\alpha$ and $C$ denote amorphous and crystalline phases.

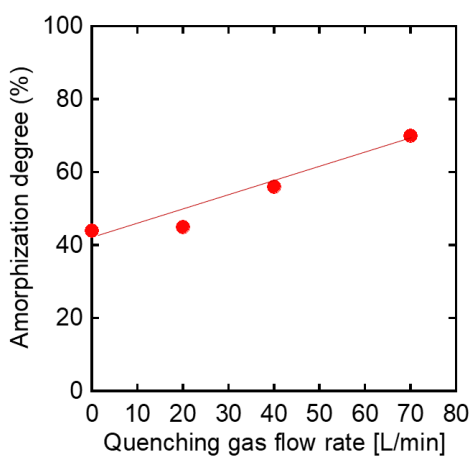

Figure 4. Effect of quenching gas flow rate on amorphization degree

Obtained result shows that higher quenching gas flow rate leads to a higher amorphization degree. There are several reasons for this phenomenon. One of the possible reason is the increased quenching rate with higher flow rate as we discussed before. However, the required quenching rate for the formation of amorphous bulks and nano materials were calculated by other researchers, which are about $5.0 \times 10^{11} \mathrm{~K} / \mathrm{s}$ (Ishimaru et al., 1997) and $7 \times 10^{9} \mathrm{~K} / \mathrm{s}$ (Shneidman, 1996), respectively. This fact suggests that the quenching rate in the current experiment is not sufficient to obtain the amorphous phase.

According to previous literatures, some researchers proved that the amorphous phase is thermodynamically preferable when the particle diameter is smaller than $5 \mathrm{~nm}$ in the case of silicon (Tomanek and Schluter, 1987). Higher quenching gas flow rate leads to larger fraction of small nanoparticles as discussed before, and that should be the reason for higher amorphization degree. The relationship between diameter of nanoparticles and amorphization degree will be discussed directly in next section.

\section{Discussion}

\subsection{Relationship between diameter of particles and amorphization degree}

Nanoparticles are divided into 4 groups with different diameters, and the volume fraction were estimated to understand the relationship between diameter and amorphization degree as shown in Figure 5. Agglomerate consists of particles with diameter less than $10 \mathrm{~nm}$ overlaps each other seriously and cannot be measured directly as shown in Figure 3. Therefore, estimation of volume for agglomerate through diameter is difficult. Here, an assumption was made that the average thickness of agglomerate is $10 \mathrm{~nm}$, and the area can be detected by TEM results directly. Then volume of agglomerate can be estimated through a simple multiplication calculation with thickness and area.

Figure 5 shows volume fraction of samples with different diameters when amorphization degree is 55\% and $86 \%$, corresponding to Figures 2 and 3, respectively. This figure presents that the volume fraction of the agglomerate is only about $11.7 \%$ in the case of lower amorphization degree, while the fraction increases to $47.4 \%$ when the amorphization degree is $86 \%$. Therefore, the higher fraction of agglomerate leads to higher amorphization degree.

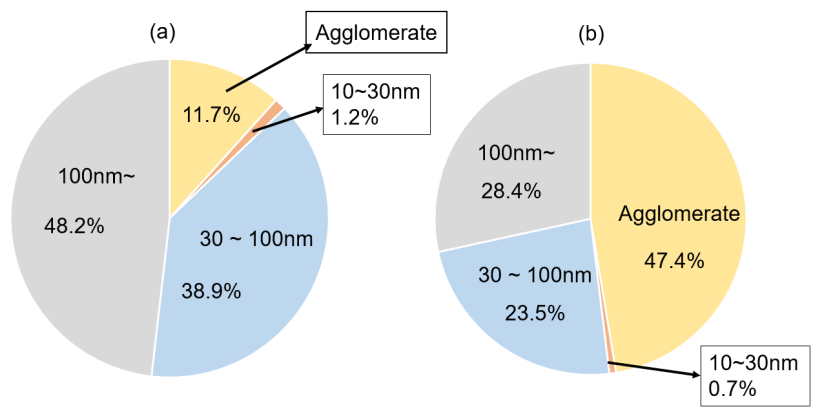

Figure 5. Volume fraction of nanoparticles with different diameters in amorphization degree is equal to (a) $55 \%$ and (b) $86 \%$

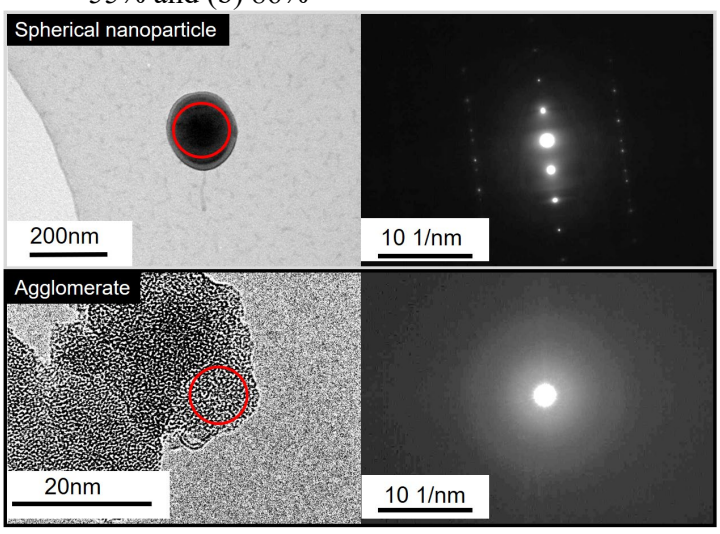

Figure 6. Diffraction patterns of different nanoparticles (red cycle is the focus of the electron beam) 
Electron diffraction analysis was conducted to understand the relationship between particular diameter and particle phase, as shown in Figure 6. For the spherical nanoparticles, some clear and regular diffraction spots can be observed in the patterns, and suggests the sample is crystal. According to the agglomerate consists of smaller particles, some diffuse rings are obtained and indicates that the agglomerate is synthesized as amorphous phase.

The reason for the higher amorphization degree can be explained through the discussion before. The higher quenching gas flow rate leads a larger amount of small nanoparticles. Those small particles are amorphous and the amorphization degree becomes higher.

\subsection{Formation mechanism of amorphous silicon nanoparticles}

Homogeneous nucleation temperatures of Si considered in the present study were estimated based on nucleation theory considering non-dimensional surface tension (Girshick et al., 1990). The homogeneous nucleation rate $J$ can be expressed as:

$$
J=\frac{\beta_{i j} n_{S}^{2} S}{12} \sqrt{\frac{\Theta}{2 \pi}} \exp \left[\Theta-\frac{4 \Theta}{27(\ln S)^{2}}\right]
$$

where $S$ is the saturation ratio and $n_{s}$ is the equilibrium saturation monomer concentration at temperature $T . \beta$ is the collision frequency function. The dimensionless surface tension $\Theta$ is given by the following equation:

$$
\Theta=\frac{\sigma s_{1}}{\mathrm{k} T}
$$

where $\sigma$ is the surface tension and $s_{1}$ is the monomer surface area. The surface tension and the saturation ratio have strong influences on determining the nucleation rate. A stable nucleation can be confirmed when the nucleation rate becomes higher than $1 \mathrm{~cm}^{-3} \mathrm{~s}^{-1}$. The corresponding saturation ratio can be defined as critical saturation ratio at nucleation temperature. Therefore, calculated nucleation temperature at the condition of this experiment is $2400 \mathrm{~K}$. Then the growth zones for the Si particles were determined, where the temperature is lower than the nucleation temperature around $2400 \mathrm{~K}$ and higher than the melting point at $1683 \mathrm{~K}$.

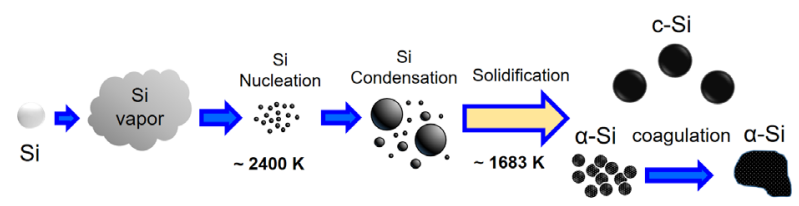

Figure 7. Formation mechanism of silicon nanoparticles

Formation mechanism of silicon nanoparticles shows in Figure 7. Firstly, silicon raw material is injected into the plasma torch, and is immediately evaporated because of the high temperature. After that, the silicon vapor goes through the center zone of the plasma and reaches the tail region where the temperature is about $2400 \mathrm{~K}$. At this time the vapor meets the condition of supersaturation and homogenous nucleation happens. Then silicon vapor condenses on the nucleus and undergoes the solidification process. The spherical nanoparticles were synthesized as crystalline with diameter larger than $10 \mathrm{~nm}$, while for the smaller silicon nanoparticles with diameter less than 10 $\mathrm{nm}$ are amorphous.

\section{Conclusion}

Amorphous silicon nanoparticles were successfully synthesized. Higher quenching gas flow rate leads to higher amorphization degree. This can be explained by the relationship between the amorphization degree and the particle diameter. Smaller diameter leads to amorphous silicon nanoparticles, while most small nanoparticles are agglomerated. This research indicates that induction thermal plasma can be used to synthesize pure amorphous material at a single step.

\section{Acknowledgements}

We would like to express our gratitude to the Ultramicroscopy Research Center for TEM observation.

\section{References}

Tomanek, D. and M. A. Schluter; "Structure and Bonding of Small Semiconductor Clusters," Physical Review B, 36, 1208-1218 (1987)

Girshick, S. L., C. P. Chiu, and P. H. McMurry; "TimeDependent Aerosol Models and Homogeneous Nucleation Rates," Aerosol Science and Technology, 13, 465-477 (1990)

Shneidman, V. A.; "Interplay of Latent Heat and TimeDependent Nucleation Effects Following Pulsed-laser Melting of a Thin Silicon Film," Journal of Applied Physics, 80, 803-811 (1996)

Ishimaru, M., S. Munetoh, and T. Motooka; "Generation of Amorphous Silicon Structures by Rapid Quenching: A Molecular-dynamics Study," Physical Review B, 56, 15133-15138 (1997)

Ng, S. H., J. Wang, D. Wexler, K. Konstantinov, Z. P. Guo, and H. K. Liu; "Highly Reversible Lithium Storage in Spheroidal Carbon-Coated Silicon Nanocomposites as Anodes for Lithiu-Ion Batteries," Angewandte Chemie International Edition, 45, 6896-6899 (2006)

Shigeta, M. and T. Watanabe; "Two-dimensional Analysis of Nanoparticle Formation in Induction Thermal Plasmas with Counterflow Cooling," Thin Solid Films, 516, 4415-4422 (2008)

Liu, X. H., L. Zhong, S Huang, S. X. Mao, T Zhu, and J. Y. Huang; "Size-dependent Fracture of Silicon Nanoparticles during Lithiation," ASC NANO, 6, 15221531 (2012)

Tanaka, M., T. Kageyama, H. Sone, S. Yoshida, D. Okamoto, and T. Watanabe; "Synthesis of Lithium Metal Oxide Nanoparticles by Induction Thermal Plasmas," Nanomaterials, 6, 60 (2016) 